\title{
FACIAL ATTENDANCE SYSTEM USING MTCNN AND FEATURE MAPPING
}

\author{
Rishabh Karmakar \\ Department of Computer Engineering \\ International Institute of Information Technology, \\ Pune, Maharashtra, India
}

\begin{abstract}
Artificial intelligence and Computer Vision are some of the fields which have shown rapid growth in recent years. These technologies have greatly reduced the effort we used to put in our day to day work and facial detection and Recognition are such revolutionary technologies. While many approaches are possible, on base, this technology works by recognizing the features of an individual face and matches it with the data which is stored in the database. Face is the representation of one's identity. Hence, we have proposed a full-fledged automated attendance system based on facial detection and recognition. Smart Attendance using Real-Time Face Recognition is a real-world solution which comes with day to day activities capable of handling everyday student / employee attendance systems. Using feature mapping, this system is capable of detecting multiple faces, saving the facial features using feature mapping and at last recognition based on the database. The Computer vision will be able to find and recognize human faces fast and precisely in images or videos that are being captured through a surveillance camera or webcam. Numerous algorithms and techniques have been developed for improving the performance of face recognition but to get the best results we have created a compounded deep learning model. Directly streaming the live video, faces will be detected, mapped and recognized and automatically saved into the database with the date and time of entry. Later the authorities can easily check the database for the complete information. Improving upon the existing systems and creating a more robust model for better and more efficient processes.
\end{abstract}

Keywords - Artificial Intelligence, Facial Recognition, Facial Detection, Multi-task Cascade Convolutional Neural Network (MTCNN), Facial Feature Mapping, Deep Learning, Computer Vision

\section{INTRODUCTION}

Traditional student attendance marking technique is often facing a lot of trouble. The face recognition student attendance system emphasizes its simplicity by eliminating classical student attendance marking techniques such as calling student names or checking respective identification cards. They not only disturb the teaching process but also cause distraction for students during exam sessions. Apart from calling names, the attendance sheet is passed around the classroom during the lecture sessions. The lecture class, especially the class with a large number of students might find it difficult to have the attendance sheet being passed around the class. Thus, face recognition student attendance systems are proposed in order to replace the manual signing of the presence of students which are burdensome and causes students to get distracted in order to sign for their attendance. Furthermore, the face recognition based automated student attendance system is able to overcome the problem of fraudulent approach and lecturers do not have to count the number of students several times to ensure the presence of the students. We just need to feed details of every student only once and afterwards the entire database of the students is maintained automatically. This facial detection and recognition decrease the manual work for humans. We are capable of image capturing from camera or real time cctv camera or even streaming video from recorder. From that offline or online data, we capture the image after that applying the face detection techniques. Face detection is detecting the face location and presence of face in images. In this face detection we see the nose, hair, ears, mouth, eyes and also different poses of faces in images.

The main objective of this project is to develop face recognition based automated student attendance systems. In order to achieve better performance, the test images and training images of this proposed approach are limited to frontal, left and upright facial images that consist of a single face only. The test images and training images have to be captured by using the same device to ensure no quality difference. In addition, the students have to register in the database to be recognized. The enrolment can be done on the spot through the user-friendly interface.

\section{MethodOLOGY}

Proposed automated attendance system can be divided into a few modules.

\section{Image Acquisition}

Image acquisition is the process by which facial scan technologies can acquire the facial feature of an individual. Various algorithms are there to detect the face of an individual from the video or image captured using a good quality camera. 


\section{International Journal of Engineering Applied Sciences and Technology, 2020 \\ Vol. 5, Issue 4, ISSN No. 2455-2143, Pages 546-550 \\ Published Online August 2020 in IJEAST (http://www.ijeast.com)}

\section{Face Detection:}

The face detection algorithm will increase the efficiency of the face recognition. There are some of the algorithms proposed for face detection as face geometry-based performances.

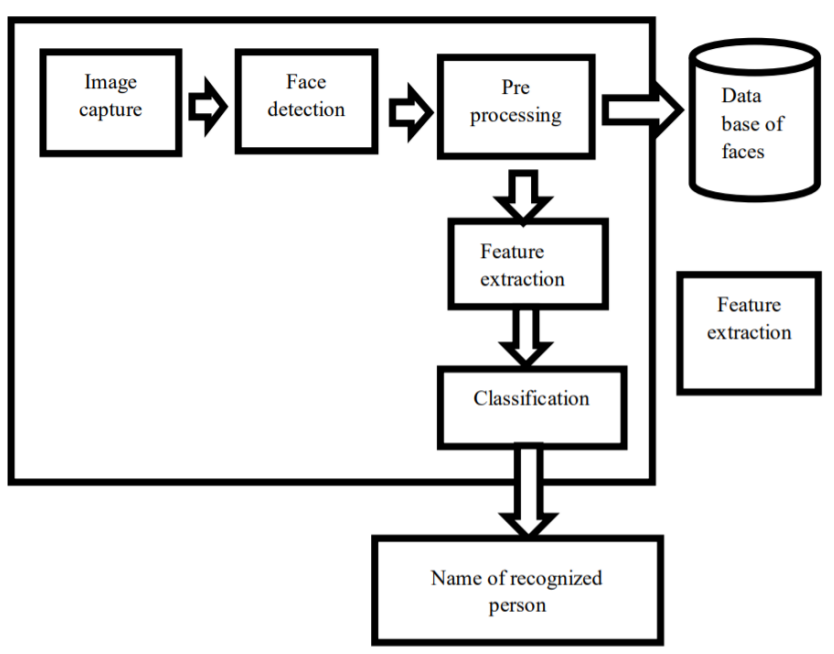

Fig. 1.Flow Chart

The observed algorithm gives the better output in different conditions to combine the multiple classifiers for better detection rate.

Later computer vision bounding boxes can be used to efficiently label them.

\section{Image Processing}

Image processing starts after successful image acquisition. In this step the image is cropped and converted to black and white format. This step facilitates initial comparison based on grey scale features of the image.

Image processing makes the acquired image ready to be compared with the image in the database. Normalization process is sometimes required to prep the image by bringing the dimension of the image similar to the saved template.

\section{Feature Extraction}

Getting the segmented facial image, we can run it through to get the facial feature map. Getting 128 relative points and then saving them. We ask the user to turn left and right to get a $128 * 3$ features of the face.

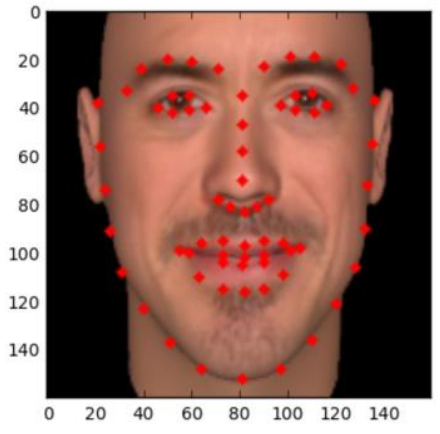

Fig. 2.Facial Feature Map

\section{Classification and Landmark Detection}

The facial recognition system attempts to match the facial features as we humans do it. There are certain features of one's face that likely to differ from one another. Some features are upper ridges of the eyes socket, distance between eyes, nose shape, width of nose, length of jaw line.

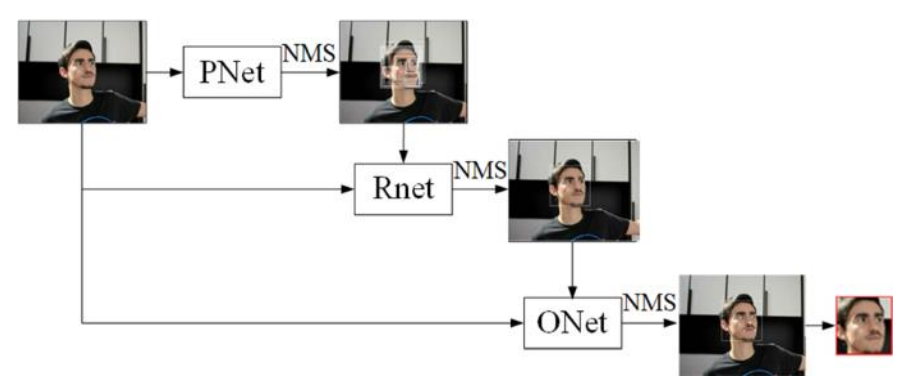

Fig. 3.Using the Neural Networks

Using the MTCNN PNet, RNet AND ONet for efficient feature extraction and landmark detection.

\section{Database Development:}

. Using a NOSQL MongoDb database to save the features of all individuals. Saving the collected landmarks of the front, left and right sides of the face of every individual.

To simply collect the attendance, something as simple as a SQL Database having the columns as ID of the individual and the appending dates marked with 'Present' or Not.

\section{Proposed Application Algorithm:}

i. The person's image will be captured.

ii. Apply pre-processing to the image.

iii. Apply detection algorithms for detecting the face and collecting features.

iv. Matching landmarks and creating the facial feature map.

v. If enrollment phase

Then store in database 


\section{International Journal of Engineering Applied Sciences and Technology, 2020 \\ Vol. 5, Issue 4, ISSN No. 2455-2143, Pages 546-550 \\ Published Online August 2020 in IJEAST (http://www.ijeast.com)}

Else check in database

And Store result in the attendance sheet

End if

\section{EXPERIMENT AND RESULT}

Rather than using the usual approach of taking a multitude of images of the user using computer vision, forming a feature map helps to greatly increase the accuracy. While in a more traditional approach, the lighting, quality or even using an accessory like the spectacles could make the user unrecognizable, all this is solved using our approach.

We are able to set the flexible confidence interval on such many facial features to be matched to be recognized.

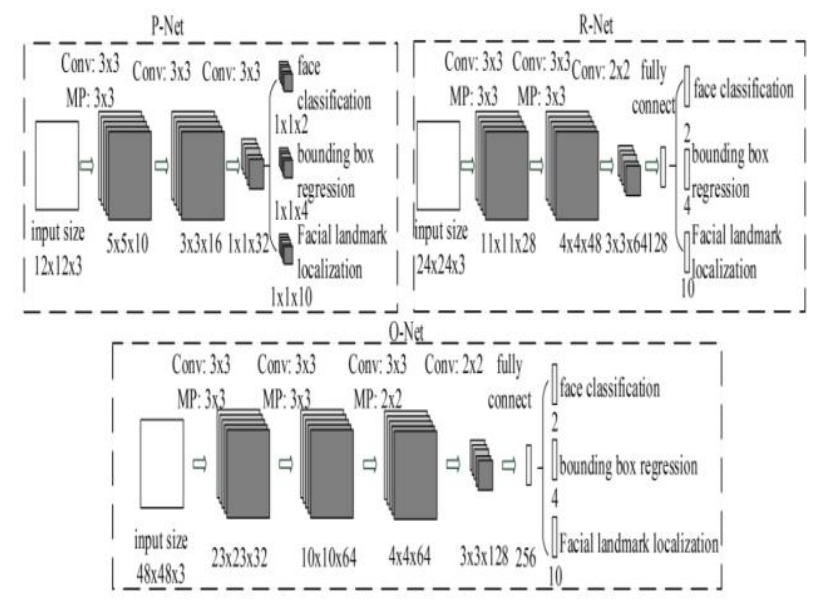

Fig. 4. The Network Architectures

Using such a compounded model structure with MTCNN building PNet, RNet and ONet as the base model and a CNN Structure in the head solidifies the accuracy and a robust system.

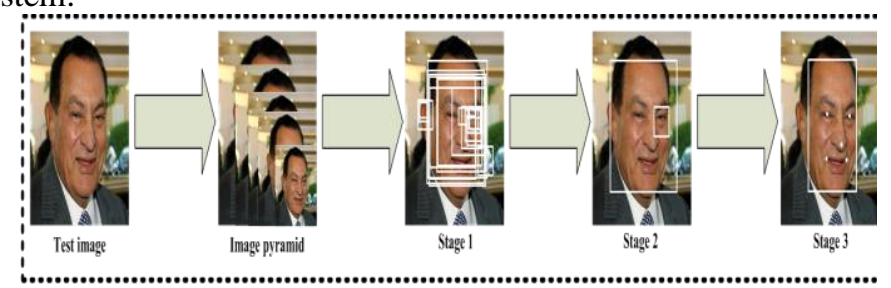

Fig. 5.Using the Convolution Neural Networks

To modify the CNN architecture, BatchNormalization and SeparableConv2D layers are notched up.

\section{a. Proposed Solution \\ We can implement various submodules for the implementation of this system.}

1. Home Page:
This contains the main interface and the various options for both the user and the teacher to browse through. Either sign up and register yourself, login or just watch the attendance sheet.

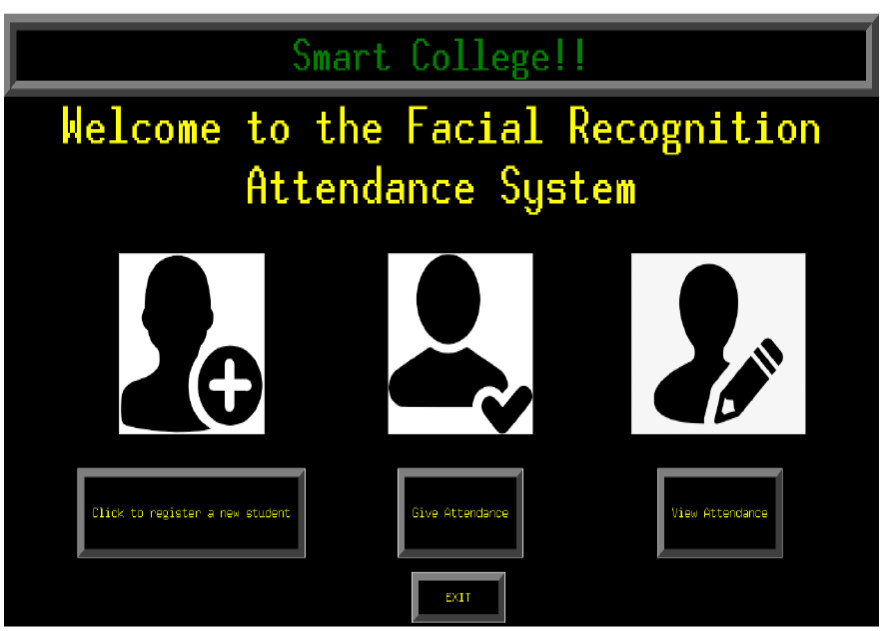

Fig. 6. The User Home Page made using Tkinter

\section{Registration}

Asking the people to see the camera and turn left and right to get features mapped on all the sides. Hence getting a $128 * 3$ landmarks for every individual.

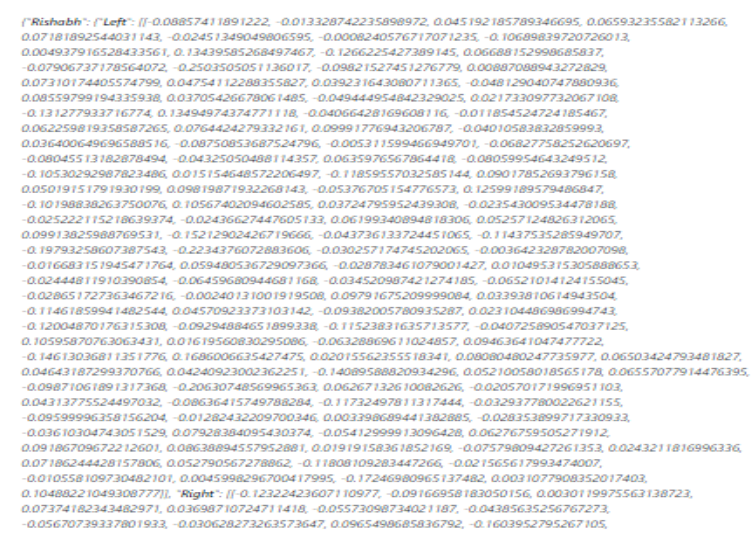

Fig. 7.Features and Landmarks

3. Giving attendance

The camera will be on all the time and the students have to just look at the camera, head up as their name is displayed and go on ahead.

Real-time processes and application of the system in a split second with the highest accuracy is achieved.

\section{Seeing the database}

The authorities can just look at the final database sheet and know the per day attendance. 


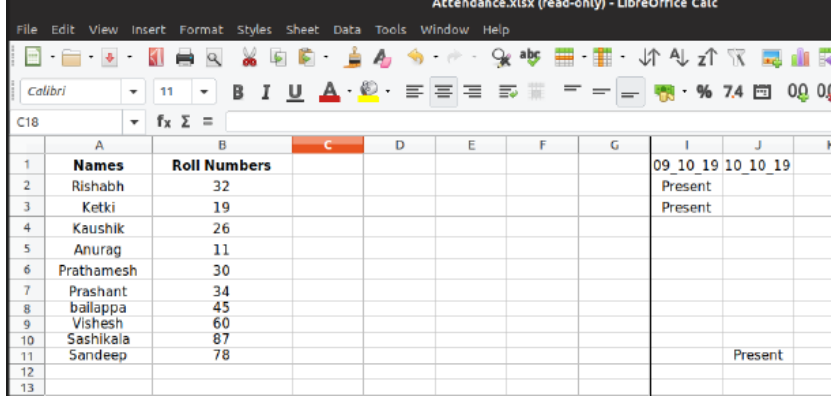

Fig. 8. Sheet with the attendance

Using a self-appending date sheet, just getting the whole attendance at one place. Any database can be used or even a spreadsheet.

\section{b. Future SCOPE}

The futuristic implementation of this Automated

Classroom Attendance System is,

01. Retina Identification and Recognition:

It is a biometric technique that uses the unique patterns on a person's retina for person identification. Retina recognition technology captures and analyzes the patterns of blood vessels on the thin nerve on the back of the eyeball that processes light entering through the pupil. Retinal patterns are highly distinctive traits. Every eye has its own totally unique pattern of blood vessels; even the eyes of identical twins are distinct. Newer, faster, better retina recognition technologies are being developed. The overall retinal scanning process may be broken down into three subprocesses: i. Image acquisition

ii. Computer based processing

iii. Features extraction and identification.

02. Automatic Speech Recognition:

Speech recognition, popularly also known as Automatic Speech Recognition (ASR) is the process of converting speech signal to a sequence of words by means of an algorithm implemented as a computer program. Speech processing is one of the major fields of signal processing. Speech recognition area aims at to develop techniques for speech input to a machine There are following building blocks for general speech recognition system:

i. Signal preprocessing

ii. Feature extraction

iii. Language model

iv. Decoder

v. Speech Recognition

03. Emotions Detection and Recognition:
Emotion detection recognition (EDR) is a method used for detection and recognition of human emotions with the incorporation of technological capabilities, such as facial recognition, speech and voice recognition, biosensing, machine learning, and pattern recognition. The algorithm will be composed of three main stages: a. Image processing stage

b. Facial feature extraction stage

c. Emotion detection stage.

In the image processing stage, the face region and facial component is extracted by using a fuzzy color filter, virtual face model, and histogram analysis method. The features for emotion detection are extracted from the facial component in the facial feature extraction stage. In the emotion detection stage, the fuzzy classifier is adopted to recognize emotion from extracted features.

\section{CONCLUSION}

Therefore, this paper successfully achieves the goal of creating a full-fledged facial recognition and attendance software and interface. Using the proposal to use the MTCNN network as the base model and a modified Convolution Neural Network $(\mathrm{CNN})$ as a head model, proves the purpose of achieving a better accuracy.

The Automated Classroom Attendance System helps in increasing the accuracy and speed ultimately achieving the high-precision real-time attendance to meet the need for automatic classroom evaluation.

\section{REFERENCE}

[1] Chaudhary Deepanshu, Rawat Akash, Maurya Deepu, Patel Ayush and Dr. Shukla Suneet (2020) "Facial Recognition Based Attendance System", International Journal of Engineering Applied Sciences and Technology, 2020 Vol. 5, Issue 1.

[2] Reddy N, Sumanth M, Babu S (2018) "A Counterpart Approach to Attendance and Feedback System using Machine Learning Techniques", Journal of Emerging Technologies and Innovative Research (JETIR), Volume 5, Issue 12.

[3] B. Yang, J. Yan, Z. Lei, and S. Z. Li, (2014) "Aggregate channel features for multi-view face detection," in IEEE International Joint Conference on Biometrics, 2014, pp. 18.

[4] S. S. Farfade, M. J. Saberian, and L. J. Li, (2015) "Multiview face detection using deep convolutional neural 
networks," in ACM on International Conference on Multimedia Retrieval, 2015, pp. 643-650.

[5] Jadhav Akshara, Jadhav Akshay, Ladhe Tushar, Krishna Yeolekar, (2017) "Automated Attendance System Using Face Recognition", International Research Journal of Engineering and Technology (IRJET), Volume 4, Issue 1.

[6] B Prabhavathi, V Tanuja, V Madhu Viswanatham and M Babu, (2017) "A smart technique for attendance system to recognize faces through parallelism", IOP Conf. Series: Materials Science and Engineering 263.

[7] Lad Prajakta, More Sonali, Parkhe Simran, Nikam Priyanka, Chaudhary Dipalee, (2017) "Student Attendance System Using Iris Detection", IJARIIE-ISSN(O)-23954396, Vol-3 Issue-2.

[8] Lukas Samuel, Mitra Aditya, Desanti Desanti, Dion Krisnadi, (2016) "Student Attendance System in Classroom Using Face Recognition Technique", Conference Paper DOI: 10.1109/ICTC.2016.7763360.

[9] M. Mathias, R. Benenson, M. Pedersoli, and L. Van Gool, (2014) "Face detection without bells and whistles," in European Conference on Computer Vision, 2014, pp. 720735.

[10] X. Zhu, and D. Ramanan, (2012) "Face detection, pose estimation, and landmark localization in the wild," in IEEE Conference on Computer Vision and Pattern Recognition, 2012, pp. 2879-2886.

[11] M. Köstinger, P. Wohlhart, P. M. Roth, and H. Bischof (2011), "Annotated facial landmarks in the wild: A largescale, real-world database for facial landmark localization," in IEEE Conference on Computer Vision and Pattern Recognition Workshops, 2011, pp. 2144-2151.

[12] P. Cappelli, P. Tambe, and V. Yakubovich et al, (Nov.2018.), "Artificial intelligence in human resources management: challenges and a path forward," SSRN Electron. J.,

[13] Liyan Zhang, Xikui Wang, Dmitri V. Kalashnikov, Sharad Mehrotra, Deva Ramanan, (2016) "Query-Driven Approach to Face Clustering and Tagging", Image Processing IEEE Transactions on, vol. 25, no. 10, pp. 4504-4513, 2016.
[14] K. S. Warke, Rupali Suralkar, Snehal Pawar, Priya Sonawane, Shraddha Wani, (2017) "A real sense based multilevel security in cloud framework using face recognition and image processing", Convergence in Technology (I2CT) 2017 2nd International Conference for, pp. 531-533, 2017.

[15] Yi-Chen Chen, Vishal M. Patel, P. Jonathon Phillips, Rama Chellappa, (2015) "Dictionary-Based Face and Person Recognition from Unconstrained Video", Access IEEE, vol. 3, pp. 1783-1798, 2015. 